\title{
ON VANISHING AND COFINITENESS OF GENERALIZED LOCAL COHOMOLOGY MODULES
}

\author{
S. H. HASSANZADEH ${ }^{1}$ AND A. VAHIDI ${ }^{2}$ \\ The second author would like to dedicate this paper \\ to the dear and loving memory of his late mother \\ who battled cancer and lost the fight in October $200 \%$.
}

\begin{abstract}
In this paper, some results on vanishing and non-vanishing of generalized local cohomology modules are presented and some relations between those modules and, Ext and ordinary local cohomology modules are studied. Also, several cofiniteness propositions for generalized local cohomology modules are established which, among other things, provide an alternative answer to a question in [Y2].
\end{abstract}

\section{INTRODUCTION}

The notion of generalized local cohomology was first introduced by J. Herzog in his habilitationss $[\mathrm{H}]$ and then continued by N. Suzuki $[\mathrm{S}]$, S. Yassemi $[\mathrm{Y}]$ and some other authors. They studied some basic duality theorems, vanishing and other properties of generalized local cohomology modules which also generalize several known facts about Ext and ordinary local cohomolgy modules.

Throughout the paper, $R$ is a commutative Noetherian ring with identity, $\mathfrak{a}$ is an ideal of $R$. For each $i \geq 0$, the generalized local cohomology functor $H_{\mathfrak{a}}^{i}(-,-)$ is defined, for all $R$-modules $M$ and $N$, by

$$
H_{\mathfrak{a}}^{i}(M, N)=\lim _{\vec{n}} \operatorname{Ext} t_{R}^{i}\left(M / \mathfrak{a}^{n} M, N\right) .
$$

In section 2, we study the vanishing and non-vanishing of generalized local cohomology modules. The crucial point is Theorem 2.2 which is interesting in itself as it provides a vanishing proposition for Ext and ordinary local cohomology modules. Then Proposition 2.3 and Corollary 2.4 show what implied from this theorem about vanishing and non-vanishing of generalized local cohomology modules. In Proposition 2.6, we extend Theorem 2.2 for Artinian property of local cohomology modules which gives a new proof for [Mel; Theorem 5.5]. Finally, in this section, Proposition 2.8 shows how the generalized local cohomology modules behave sharply at the non-vanishing bounds for Ext and local cohomology modules. We also apply this proposition in section 3.

In section 3, we study the cofiniteness property of generalized local cohomology modules. In the first proposition we show that for finite $R$-modules $M$ and $N$ with $p d(M)<\infty$, the $R$-module

2000 Mathematics Subject Classification. 13D45, 14B15.

Key words and phrases. Generalized local cohomology, Cofiniteness, Spectral sequence. 
$H_{\mathfrak{a}}^{p d(M)+\operatorname{dim}(N)}(M, N)$ is not only Artinian, as shown by A. Mafi [M; Theorem 2.9], but also acofinite. Then in Proposition 3.4 we generalize [Mel; Corollary 2.5] for a Serre subcategory of the category of $R$-modules. Furthermore we generalize and give a new proof to [Mel; Corollary $3.14]$ for arbitrary $R$-modules in Proposition 3.5. These two propositions can also be employed to prove Proposition 3.6 which presents some finite generalized local cohomology modules. The main theorem of section 3 is Theorem 3.7 which provides some sufficient conditions for generalized local cohomology modules to be $\mathfrak{a}$-cofinite. Even though the assumptions in this theorem are apparently strong, they are practically useful as we can see in the next corollaries which demonstrate some new facts and represent some older facts about $\mathfrak{a}$-cofiniteness of generalized local cohomology modules.

\section{VANISHING AND NON-VANISHING RESULTS}

In this section, we investigate some basic properties of generalized local cohomology modules. Most properties of generalized local cohomology modules inherit from the spectral sequence

$$
E_{2}^{p, q}:=\operatorname{Ext}_{R}^{p}\left(M, H_{\mathfrak{a}}^{q}(X)\right) \underset{p}{\longrightarrow} H_{\mathfrak{a}}^{p+q}(M, X)
$$

in particular, when we put this spectral sequence in a double complex. All of the facts in this section can be proved by means of the method that we apply in Theorem 2.2 but to shorten the other proofs, we just mention the corresponding spectral sequence's property.

The following lemma is an immediate consequence of $(*)$ or can be drawn directly, applying the basic properties of generalized local cohomology such as [DST; Lemma 2.1(i)].

Lemma 2.1. Suppose that $M$ is a finite $R$-module and that $X$ is an arbitrary $R$-module. Then

(i) $\Gamma_{\mathfrak{a}}(M, X)=0$ whenever $\Gamma_{\mathfrak{a}}(X)=0$.

(ii) $\Gamma_{\mathfrak{a}}(M, X)=H_{\mathfrak{a}}^{1}(M, X)=0$ whenever $\Gamma_{\mathfrak{a}}(X)=H_{\mathfrak{a}}^{1}(X)=0$.

Theorem 2.2. Let $X$ be an arbitrary $R$-module (not necessarily finite) and $t$ be a positive integer. Then the following statements are equivalent:

(i) $H_{\mathfrak{a}}^{i}(X)=0$ for all $0 \leq i<t$.

(ii) $\operatorname{Ext}_{R}^{i}(R / \mathfrak{a}, X)=0$ for all $0 \leq i<t$.

Proof. We prove by using induction on t. Let $t=1$. Consider the isomorphism $\operatorname{Hom}_{R}(R / \mathfrak{a}, X) \cong$ $\operatorname{Hom}_{R}\left(R / \mathfrak{a}, \Gamma_{\mathfrak{a}}(X)\right)$; so that we have an equivalent condition that $\operatorname{Hom}_{R}(R / \mathfrak{a}, X)=0$ if and only if $\Gamma_{\mathfrak{a}}(X)=0$, since $\operatorname{Supp}\left(\Gamma_{\mathfrak{a}}(X)\right) \subseteq V(\mathfrak{a})$. Hence the assertion holds in this case.

Suppose that $t>1$ and that we have proved the theorem for $t-1$ and all $R$-modules $X$. Let $Y$ be an $R$-module and consider the exact sequence

$$
0 \rightarrow Y \rightarrow E(Y) \rightarrow L \rightarrow 0
$$


where $E(Y)$ is an injective hull of $Y$ and $L$ is an $R$-module. Applying the derived functors of $\operatorname{Hom}_{R}(R / \mathfrak{a},-)$ and $\Gamma_{\mathfrak{a}}(-)$ to the above short exact sequence, we have exact sequences and isomorphisms:

$$
\begin{gathered}
0 \rightarrow \operatorname{Hom}_{R}(R / \mathfrak{a}, Y) \rightarrow \operatorname{Hom}_{R}(R / \mathfrak{a}, E(Y)) \rightarrow \operatorname{Hom}_{R}(R / \mathfrak{a}, L) \rightarrow \operatorname{Ext}_{R}^{1}(R / \mathfrak{a}, Y) \rightarrow 0, \\
\operatorname{Ext}_{R}^{i-1}(R / \mathfrak{a}, L) \cong \operatorname{Ext}_{R}^{i}(R / \mathfrak{a}, Y)
\end{gathered}
$$

for all $i>1$, and

$$
\begin{aligned}
0 \rightarrow \Gamma_{\mathfrak{a}}(Y) \rightarrow \Gamma_{\mathfrak{a}}(E(Y)) & \rightarrow \Gamma_{\mathfrak{a}}(L) \rightarrow H_{\mathfrak{a}}^{1}(Y) \rightarrow 0, \\
H_{\mathfrak{a}}^{i-1}(L) & \cong H_{\mathfrak{a}}^{i}(Y)
\end{aligned}
$$

for all $i>1$.

Now, assume that $H_{\mathfrak{a}}^{i}(Y)=0$ for all $0 \leq i<t$. Since $\Gamma_{\mathfrak{a}}(Y)=0$, we have $\Gamma_{\mathfrak{a}}(E(Y))=0$ from the fact that $E(Y)$ is an essential extension of $Y$; so that $\operatorname{Hom}_{R}(R / \mathfrak{a}, Y) \subseteq H_{R}(R / \mathfrak{a}, E(Y))=0$. Hence we have, by the above isomorphisms and exact sequences, $R$-isomorphisms:

$$
\operatorname{Ext}_{R}^{i-1}(R / \mathfrak{a}, L) \cong \operatorname{Ext}_{R}^{i}(R / \mathfrak{a}, Y) \quad \text { and } \quad H_{\mathfrak{a}}^{i-1}(L) \cong H_{\mathfrak{a}}^{i}(Y)
$$

for all $i>0$.

Now, $H_{\mathfrak{a}}^{i}(L)=0$ for all $0 \leq i<t-1$ form the latter isomorphisms. Hence $\operatorname{Ext}_{R}^{i}(R / \mathfrak{a}, L)=0$ for all $0 \leq i<t-1$ by the induction hypothesis on $L$. Again from the above isomorphisms, $\operatorname{Ext}_{R}^{i}(R / \mathfrak{a}, Y)=0$ for all $0 \leq i<t$.

Conversely, as the above proof, we see that if $\operatorname{Ext}_{R}^{i}(R / \mathfrak{a}, Y)=0$ for all $0 \leq i<t$, then $H_{\mathfrak{a}}^{i}(Y)=0$ for all $0 \leq i<t$. We leave the proof to the reader. The proof is completed.

Proposition 2.3. Let $M$ be a finitely generated $R$-module, $X$ be an $R$-module, and $t$ be a positive integer. If $R$-module $X$ satisfies the equivalent conditions in Theorem 2.2 for the integer $t$, then we have

(i) $H_{\mathfrak{a}}^{i}(M, X)=0$ for all $0 \leq i<t$.

(ii) $H_{\mathfrak{a}}^{t}(M, X) \cong \operatorname{Hom}_{R}\left(M, H_{\mathfrak{a}}^{t}(X)\right)$.

(iii) $\operatorname{Ext}_{R}^{t}(R / \mathfrak{a}, X) \cong \operatorname{Hom}_{R}\left(R / \mathfrak{a}, H_{\mathfrak{a}}^{t}(X)\right)$.

Proof. Before beginning the proof, recall that by $D_{\mathfrak{a}}(X)$ we mean the ideal transform of $X$ with respect to $\mathfrak{a}$ which is defined by $D_{\mathfrak{a}}(X):=\lim _{\vec{n}} \operatorname{Hom}_{R}\left(\mathfrak{a}^{n}, X\right)$. For the basic definitions and theorems about local cohomology and ideal transform we refer the reader to [BS].

We use induction on $t$. Let $t=1$. Since $\Gamma_{\mathfrak{a}}(X)=0, \Gamma_{\mathfrak{a}}(M, X)=0$ by Lemma 2.1(i). We have $\Gamma_{\mathfrak{a}}\left(D_{\mathfrak{a}}(X)\right)=H_{\mathfrak{a}}^{1}\left(D_{\mathfrak{a}}(X)\right)=0$ by [BS; Corollary 2.2.8(iv)]. Therefore $\Gamma_{\mathfrak{a}}\left(M, D_{\mathfrak{a}}(X)\right)=$ $H_{\mathfrak{a}}^{1}\left(M, D_{\mathfrak{a}}(X)\right)=0$ by Lemma 2.1(ii) and, $\operatorname{Hom}_{R}\left(R / \mathfrak{a}, D_{\mathfrak{a}}(X)\right)=\operatorname{Ext}_{R}^{1}\left(R / \mathfrak{a}, D_{\mathfrak{a}}(X)\right)=0$ by Theorem 2.2; so that the ideal transform sequence $0 \rightarrow X \rightarrow D_{\mathfrak{a}}(X) \rightarrow H_{\mathfrak{a}}^{1}(X) \rightarrow 0$, yields $\operatorname{Ext}_{R}^{1}(R / \mathfrak{a}, X) \cong \operatorname{Hom}_{R}\left(R / \mathfrak{a}, H_{\mathfrak{a}}^{1}(X)\right)$ and $H_{\mathfrak{a}}^{1}(M, X) \cong \Gamma_{\mathfrak{a}}\left(M, H_{\mathfrak{a}}^{1}(X)\right)$. Now, the latter isomorphisms in conjunction with [YKS; Lemma 1.1] imply the assertion in the case where $t=1$. 
Let $Y$ be an $R$-module and $t>1$. Also suppose that our claims are satisfied for $t-1$ and all $R$-modules $X$.

Now, assume that $H_{\mathfrak{a}}^{i}(Y)=0$ for all $0 \leq i<t$. Since $\Gamma_{\mathfrak{a}}(Y)=0$, we have $\Gamma_{\mathfrak{a}}(E(Y))=0$ from the fact that $E(Y)$ is an essential extension of $Y$; so that $\Gamma_{\mathfrak{a}}(M, E(Y))=0$ by Lemma 2.1(i). Using the exact sequence $0 \rightarrow Y \rightarrow E(Y) \rightarrow L \rightarrow 0$ as we used in the above theorem and applying the derived functors of $\operatorname{Hom}_{R}(R / \mathfrak{a},-), \Gamma_{\mathfrak{a}}(M,-)$ and $\Gamma_{\mathfrak{a}}(-)$ to this short exact sequence, we obtain the isomorphisms:

$$
\operatorname{Ext}_{R}^{i-1}(R / \mathfrak{a}, L) \cong \operatorname{Ext}_{R}^{i}(R / \mathfrak{a}, Y), H_{\mathfrak{a}}^{i-1}(M, L) \cong H_{\mathfrak{a}}^{i}(M, Y) \text { and } H_{\mathfrak{a}}^{i-1}(L) \cong H_{\mathfrak{a}}^{i}(Y)
$$

for all $i>0$.

Now, our assumption on $Y$ and the latter isomorphisms show that, $H_{\mathfrak{a}}^{i}(L)=0$ for all $0 \leq i<$ $t-1$. Hence $H_{\mathfrak{a}}^{t-1}(M, L) \cong \operatorname{Hom}_{R}\left(M, H_{\mathfrak{a}}^{t-1}(L)\right), \operatorname{Ext}_{R}^{t-1}(R / \mathfrak{a}, L) \cong \operatorname{Hom}_{R}\left(R / \mathfrak{a}, H_{\mathfrak{a}}^{t-1}(L)\right)$ and $H_{\mathfrak{a}}^{i}(M, L)=0$ for all $0 \leq i<t-1$ by the induction hypothesis on $L$. Again from the above isomorphisms, we have $H_{\mathfrak{a}}^{t}(M, Y) \cong \operatorname{Hom}_{R}\left(M, H_{\mathfrak{a}}^{t}(Y)\right), \operatorname{Ext}_{R}^{t}(R / \mathfrak{a}, Y) \cong \operatorname{Hom}_{R}\left(R / \mathfrak{a}, H_{\mathfrak{a}}^{t}(Y)\right)$ and $H_{\mathfrak{a}}^{i}(M, Y)=0$ for all $0 \leq i<t$, as we desired.

The following corollary can be obtained, by straightforward applications, from Theorem 2.2 and Proposition 2.3.

Corollary 2.4. Let $M, N$ be non-zero finite $R$-modules, $X$ be an arbitrary $R$-module, and $t$ be a positive integer. Then

(i) (a) $\operatorname{Ext}_{R}^{1}\left(R / \mathfrak{a}, D_{\mathfrak{a}}(X)\right)=H_{\mathfrak{a}}^{1}\left(M, D_{\mathfrak{a}}(X)\right)=0$.

(b) $\operatorname{Ext}_{R}^{2}\left(R / \mathfrak{a}, D_{\mathfrak{a}}(X)\right) \cong \operatorname{Hom}_{R}\left(R / \mathfrak{a}, H_{\mathfrak{a}}^{2}(X)\right)$ and $H_{\mathfrak{a}}^{2}\left(M, D_{\mathfrak{a}}(X)\right) \cong \operatorname{Hom}_{R}\left(M, H_{\mathfrak{a}}^{2}(X)\right)$.

(c) $\operatorname{Ext}_{R}^{1}\left(R / \mathfrak{a}, X / \Gamma_{\mathfrak{a}}(X)\right) \cong \operatorname{Hom}_{R}\left(R / \mathfrak{a}, H_{\mathfrak{a}}^{1}(X)\right)$ and $H_{\mathfrak{a}}^{1}\left(M, X / \Gamma_{\mathfrak{a}}(X)\right) \cong \operatorname{Hom}_{R}\left(M, H_{\mathfrak{a}}^{1}(X)\right)$.

(ii) If $H_{\mathfrak{a}}^{i}(X)=0$ for all $0 \leq i<t$, then $H_{\mathfrak{a}}^{i}(M, X)=0$ for all $0 \leq i<t$.

In this case $H_{\mathfrak{a}}^{t}(M, X) \neq 0$ if and only if $\operatorname{Supp}(M) \cap \operatorname{Ass}\left(H_{\mathfrak{a}}^{t}(X)\right) \neq \varnothing$.

(iii) If a maximal ideal $\mathfrak{m}$ belongs to $\operatorname{Supp}(M)$ and $t=\operatorname{grade}(\mathfrak{m}, N)$, then $H_{\mathfrak{m}}^{t}(M, N) \neq 0$.

(iv) (c.f. [S; Theorem 2.3]) If $(R, \mathfrak{m})$ is a local ring and $t=\operatorname{depth}(N)$, then $H_{\mathfrak{m}}^{t}(M, N) \neq 0$.

Lemma 2.5. Suppose that $P$ is a finite projective $R$-module and $X$ is an arbitrary $R$-module. Then $H_{\mathfrak{a}}^{i}(P, X) \cong \operatorname{Hom}_{R}\left(P, H_{\mathfrak{a}}^{i}(X)\right)$ for all $i \geq 0$.

Proof. This lemma can be proved by using the method implied in Proposition 2.3. It is also an immediate consequence of the spectral sequence

$$
E_{2}^{i, j}:=\operatorname{Ext}_{R}^{i}\left(P, H_{\mathfrak{a}}^{j}(X)\right) \underset{i}{\Longrightarrow} H_{\mathfrak{a}}^{i+j}(P, X)
$$

where in the corresponding double complex $\left(E_{2}^{i, j}\right)_{i \geq 0, j \geq 0}$ all of the columns except the first one vanish. 
In the course of the remaining parts of the paper for an arbitrary $R$-module $X$, by $\operatorname{cd}(\mathfrak{a}, X)$ (cohomological dimension of $X$ at $\mathfrak{a}$ ) we mean the largest integer $i$ in which $H_{\mathfrak{a}}^{i}(X)$ is non-zero. Also $p d(X)$ and $\operatorname{dim}(X)$ are denoted as the projective dimension and the Krull dimension of $X$, respectively. The next proposition shows how Artinian property behaves similarly at the initial points of Ext and at those of ordinary local cohomology modules. This proposition gives a new proof for [Mel; Theorem 5.5].

Proposition 2.6. Let $X$ be an arbitrary $R$-module and $t$ be a positive integer. Then the following statements are equivalent:

(i) $H_{\mathfrak{a}}^{i}(X)$ is Artinian for all $0 \leq i<t$.

(ii) $\operatorname{Ext}_{R}^{i}(R / \mathfrak{a}, X)$ is Artinian for all $0 \leq i<t$.

Proof. We prove by induction on $t$. Considering $0: X \mathfrak{a}=0:_{\Gamma_{\mathfrak{a}}(X)} \mathfrak{a}$ and applying Melkersson's Theorem [BS; Theorem 7.1.2], the case where $t=1$ is straightforward.

Let $Y$ be an $R$-module and $t>1$. Also assume that we have proved the claim for $t-1$ and all $R$-modules $X$. Let $N=Y / \Gamma_{\mathfrak{a}}(Y)$ and $L=E(N) / N$ where $E(N)$ is an injective hull of $N$. Since $\Gamma_{\mathfrak{a}}(N)=0$, we have $\Gamma_{\mathfrak{a}}(E(N))=0$ from the fact that $E(N)$ is an essential extension of $N$. Now consider the exact sequence

$$
0 \rightarrow N \rightarrow E(N) \rightarrow L \rightarrow 0 .
$$

Applying the derived functors of $\operatorname{Hom}_{R}(R / \mathfrak{a},-)$ and $\Gamma_{\mathfrak{a}}(-)$ to the above short exact sequence, we have isomorphisms:

$$
\operatorname{Ext}_{R}^{i-1}(R / \mathfrak{a}, L) \cong \operatorname{Ext}_{R}^{i}(R / \mathfrak{a}, N) \quad \text { and } \quad H_{\mathfrak{a}}^{i-1}(L) \cong H_{\mathfrak{a}}^{i}(N)
$$

for all $i>0$.

Now, assume that $H_{\mathfrak{a}}^{i}(Y)$ is Artinian for all $0 \leq i<t$. From the latter isomorphisms and [BS; Corollary 2.1.7], we have $H_{\mathfrak{a}}^{i}(L)$ is Artinian for all $0 \leq i<t-1$. Hence $\operatorname{Ext}_{R}^{i}(R / \mathfrak{a}, L)$ is Artinian for all $0 \leq i<t-1$ by the induction hypothesis on $L$. Again by the above isomorphisms, $\operatorname{Ext}_{R}^{i}(R / \mathfrak{a}, N)$ is Artinian for all $0 \leq i<t$. Now, the exact sequence $0 \rightarrow \Gamma_{\mathfrak{a}}(Y) \rightarrow Y \rightarrow N \rightarrow 0$ in conjunction with Artinianness of $\Gamma_{\mathfrak{a}}(Y)$ show that, $\operatorname{Ext}_{R}^{i}(R / \mathfrak{a}, Y)$ is Artinian for all $0 \leq i<t$.

Conversely, as the above proof, we see that if $\operatorname{Ext}_{R}^{i}(R / \mathfrak{a}, Y)$ is Artinian for all $0 \leq i<t$, then $H_{\mathfrak{a}}^{i}(Y)$ is Artinian for all $0 \leq i<t$. We leave the proof to the reader. The proof is completed.

Proposition 2.7. Let $M$ be a finite $R$-module with $p d(M)<\infty, X$ be an arbitrary $R$-module, and $t$ be a positive integer. If $R$-module $X$ satisfies the equivalent conditions in Proposition 2.6 for the integer $t$, then $H_{\mathfrak{a}}^{i}(M, X)$ is Artinian for all $0 \leq i<t$.

Proof. We use induction on $p d(M)$. The case where $p d(M)=0$ yields $M \oplus M^{\prime} \cong R^{n}$ for some $R$-module $M^{\prime}$ and some integer $n$. Then, by Lemma 2.5,

$$
H_{\mathfrak{a}}^{i}(M, X) \oplus H_{\mathfrak{a}}^{i}\left(M^{\prime}, X\right) \cong \operatorname{Hom}_{R}\left(M, H_{\mathfrak{a}}^{i}(X)\right) \oplus \operatorname{Hom}_{R}\left(M^{\prime}, H_{\mathfrak{a}}^{i}(X)\right)
$$




$$
\cong \operatorname{Hom}_{R}\left(R^{n}, H_{\mathfrak{a}}^{i}(X)\right) \cong H_{\mathfrak{a}}^{i}(X)^{n}
$$

which gives the assertion in this case.

Suppose that $p d(M)>0$ and that the assertion is true for every finite $R$-module $T$ with $p d(T)<$ $p d(M)$. Taking the exact sequence $0 \rightarrow T \rightarrow F \rightarrow M \rightarrow 0$, where $F$ is free and $T$ is an $R$-module, we set the long exact sequence

$$
\cdots \rightarrow H_{\mathfrak{a}}^{i-1}(T, X) \rightarrow H_{\mathfrak{a}}^{i}(M, X) \rightarrow H_{\mathfrak{a}}^{i}(F, X) \rightarrow \cdots
$$

where by induction hypothesis $H_{\mathfrak{a}}^{i-1}(T, X)$ and $H_{\mathfrak{a}}^{i}(F, X)$ are Artinian; so that $H_{\mathfrak{a}}^{i}(M, X)$ is Artinian as desired.

The below proposition can also be proved either by considering the spectral sequence $(*)$ or using the method in Proposition 2.3 based on an induction on $c d(\mathfrak{a}, X)$.

Proposition 2.8. (compare $(Y$; Theorem 2.5]) Let $M$ be a finite R-module with $p d(M)<\infty$ and $X$ be an arbitrary $R$-module. Then for all $i>p d(M)+c d(\mathfrak{a}, X), H_{\mathfrak{a}}^{i}(M, X)=0$ and

$$
H_{\mathfrak{a}}^{p d(M)+c d(\mathfrak{a}, X)}(M, X) \cong \operatorname{Ext}_{R}^{p d(M)}\left(M, H_{\mathfrak{a}}^{c d(\mathfrak{a}, X)}(X)\right) .
$$

\section{Cofiniteness Results}

In this section we deal with the cofiniteness property of generalized local cohomology modules. Recall that an $R$-module $X$ is said to be $\mathfrak{a}$-cofinite if $\operatorname{Supp}(X) \subseteq V(\mathfrak{a})$ and either $\operatorname{Ext}_{R}^{i}(R / \mathfrak{a}, X)$ is finite for all $i$ or equally, $\operatorname{Tor}_{i}^{R}(R / \mathfrak{a}, X)$ is finite for all $i$ [Mel; Theorem 2.1].

Proposition 3.1. (compare [M; Theorem 2.9)]) Assume that $M, N$ are finite $R$-modules such that $t=p d(M)$ and $d=\operatorname{dim}(N)$ are finite. Then $H_{\mathfrak{a}}^{t+d}(M, N)$ is an $\mathfrak{a}$-cofinite Artinian module.

Proof. If $c d(\mathfrak{a}, N)<d, H_{\mathfrak{a}}^{t+d}(M, N)=0$ by Proposition 2.8. Hence suppose that $c d(\mathfrak{a}, N)=d$ then we have $H_{\mathfrak{a}}^{t+d}(M, N) \cong \operatorname{Ext}_{R}^{t}\left(M, H_{\mathfrak{a}}^{d}(N)\right)$, again by Proposition 2.8. Now, according to [Mel; Proposition 5.1] $H_{\mathfrak{a}}^{d}(N)$ is an $\mathfrak{a}$-cofinite Artinian module and also by [Mel; Corollary 4.4] the category of $\mathfrak{a}$-cofinite Artinian modules is a Serre subcategory of the category of $R$-modules, i.e. this subcategory is closed under taking subobjects, quotients and extensions. Therefore $\operatorname{Ext}_{R}^{t}\left(M, H_{\mathfrak{a}}^{d}(N)\right)$ and hence $H_{\mathfrak{a}}^{t+d}(M, N)$ is an $\mathfrak{a}$-cofinite Artinian module.

Notation 3.2. For an ideal $\mathfrak{b}$ of $R$, we denote the category of finite $R / \mathfrak{b}$-modules by $\mathcal{C}^{f}(R / \mathfrak{b})$ and the category of finite $R$-modules whose support contained in $V(\mathfrak{b})$ by $\mathcal{C}^{\mathfrak{b}}(R)$. Also we denote the category of $R$-modules by $\mathcal{C}(R)$.

Considering the fact that each member of $\mathcal{C}^{\mathfrak{a}}(R)$ belongs to $\mathcal{C}^{f}\left(R / \mathfrak{a}^{n}\right)$ for some positive integer $n$ and appling a straightforward induction on $n$, we deduce the following useful lemma.

Lemma 3.3. Suppose that $T$ is a middle exact covariant (contravariant) functor from $\mathcal{C}(R)$ to $\mathcal{C}(R)$ and that $\delta$ is a Serre subcategory of $\mathcal{C}(R)$. Then $T(L) \in \delta$ for each $L \in \mathcal{C}^{\mathfrak{a}}(R)$ whenever $T(L) \in \delta$ for each $L \in \mathcal{C}^{f}(R / \mathfrak{a})$. 
Proposition 3.4. (compare [Mel; Corollary 2.5]) Let $X$ be an arbitrary $R$-module, $t$ be a nonnegative integer, and $\delta$ be a Serre subcategory of $\mathcal{C}(R)$. Suppose $\mathfrak{b}$ be a second ideal of $R$ contains a. Then

(i) If $\operatorname{Ext}_{R}^{i}(R / \mathfrak{a}, X) \in \delta$ for all $0 \leq i \leq t$, then $\operatorname{Ext}_{R}^{i}(N, X) \in \delta$ for all $0 \leq i \leq t$ and all $N \in \mathcal{C}^{\mathfrak{a}}(R)$. In particular, $\operatorname{Ext}_{R}^{i}(R / \mathfrak{b}, X) \in \delta$ for all $0 \leq i \leq t$.

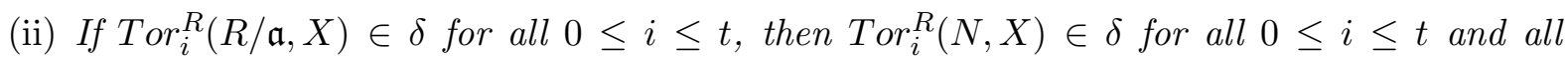
$N \in \mathcal{C}^{\mathfrak{a}}(R)$. In particular, $\operatorname{Tor}_{i}^{R}(R / \mathfrak{b}, X) \in \delta$ for all $0 \leq i \leq t$.

Proof. We just prove (i), the proof for (ii) is similar. First, we use induction on $i$ to prove that $\operatorname{Ext}_{R}^{i}(N, X) \in \delta$ whenever $N \in \mathcal{C}^{f}(R / \mathfrak{a})$. Let $i=0$ and $N \in \mathcal{C}^{f}(R / \mathfrak{a})$. Considering a suitable integer $s$ and an exact sequence $(R / \mathfrak{a})^{s} \rightarrow N \rightarrow 0$, we obtain the exact sequence $0 \rightarrow \operatorname{Hom}_{R}(N, X) \rightarrow \operatorname{Hom}_{R}\left((R / \mathfrak{a})^{s}, X\right)$ where $\operatorname{Hom}_{R}\left((R / \mathfrak{a})^{s}, X\right) \in \delta$ by our assumption; so that $H_{\operatorname{Hom}}(N, X) \in \delta$ which completes the proof for the case where $i=0$.

Suppose that $1 \leq i \leq t$ and that our assertion holds for $i-1$. Let $N \in \mathcal{C}^{f}(R / \mathfrak{a})$. By an exact sequence $0 \rightarrow K \rightarrow(R / \mathfrak{a})^{s} \rightarrow N \rightarrow 0$, where $K \in \mathcal{C}^{f}(R / \mathfrak{a})$ and $s$ is an integer, we obtain a long exact sequence

$$
\cdots \rightarrow \operatorname{Ext}_{R}^{i-1}(K, X) \rightarrow \operatorname{Ext}_{R}^{i}(N, X) \rightarrow \operatorname{Ext}_{R}^{i}\left((R / \mathfrak{a})^{s}, X\right) \rightarrow \cdots
$$

where by the assumption in (i), $\operatorname{Ext}_{R}^{i}\left((R / \mathfrak{a})^{s}, X\right) \in \delta$ and by induction hypothesis $\operatorname{Ext}_{R}^{i-1}(K, X) \in \delta$ therefore $\operatorname{Ext}_{R}^{i}(N, X) \in \delta$. This terminates the induction argument. Now, an application of Lemma 3.3 completes the proof.

Proposition 3.5. (compare [Mel; Corollary 3.14]) Suppose that $X$ is an arbitrary $R$-module and that $\operatorname{cd}(\mathfrak{a}, X)=1$. Then for all $i \geq 0, \operatorname{Ext}_{R}^{i}\left(R / \mathfrak{a}, H_{\mathfrak{a}}^{1}(X)\right) \cong \operatorname{Ext}_{R}^{i+1}\left(R / \mathfrak{a}, X / \Gamma_{\mathfrak{a}}(X)\right)$. In particular, if $X$ is finite, then $H_{\mathfrak{a}}^{i}(X)$ is $\mathfrak{a}$-cofinite for all $i$.

Proof. Since $c d(\mathfrak{a}, X)=1, H_{\mathfrak{a}}^{i}\left(D_{\mathfrak{a}}(X)\right)=0$ for all $i \geq 0$. Hence $\operatorname{Ext}_{R}^{i}\left(R / \mathfrak{a}, D_{\mathfrak{a}}(X)\right)=0$ for all $i \geq 0$ by Theorem 2.2. Now, by the ideal transform sequence

$$
0 \rightarrow X / \Gamma_{\mathfrak{a}}(X) \rightarrow D_{\mathfrak{a}}(X) \rightarrow H_{\mathfrak{a}}^{1}(X) \rightarrow 0,
$$

we get $\operatorname{Ext}_{R}^{i}\left(R / \mathfrak{a}, H_{\mathfrak{a}}^{1}(X)\right) \cong \operatorname{Ext}_{R}^{i+1}\left(R / \mathfrak{a}, X / \Gamma_{\mathfrak{a}}(X)\right)$ for all $i \geq 0$.

The next proposition introduces some finite generalized local cohomology modules.

Proposition 3.6. Suppose that $M, N$ are finite $R$-modules such that $M=\Gamma_{\mathfrak{a}}(M)$ and $c d(\mathfrak{a}, N)=1$. Then $H_{\mathfrak{a}}^{i}(M, N)$ is finite for all $i \geq 0$.

Proof. Since $\operatorname{cd}(\mathfrak{a}, N)=1, H_{\mathfrak{a}}^{i}\left(D_{\mathfrak{a}}(N)\right)=0$ for all $i \geq 0$. Hence $H_{\mathfrak{a}}^{i}\left(M, D_{\mathfrak{a}}(N)\right)=0$ whenever $i \geq 0$ by Proposition 2.3. Then the ideal transform sequence

$$
0 \rightarrow N / \Gamma_{\mathfrak{a}}(N) \rightarrow D_{\mathfrak{a}}(N) \rightarrow H_{\mathfrak{a}}^{1}(N) \rightarrow 0
$$


in conjunction with [YKS; Lemma 1.1] yield the isomorphisms below: $H_{\mathfrak{a}}^{i+1}\left(M, N / \Gamma_{\mathfrak{a}}(N)\right) \cong H_{\mathfrak{a}}^{i}\left(M, H_{\mathfrak{a}}^{1}(N)\right) \cong \operatorname{Ext}_{R}^{i}\left(M, H_{\mathfrak{a}}^{1}(N)\right)$ and $H_{\mathfrak{a}}^{i}\left(M, \Gamma_{\mathfrak{a}}(N)\right) \cong \operatorname{Ext}_{R}^{i}\left(M, \Gamma_{\mathfrak{a}}(N)\right)$ for all $i \geq 0$. By Proposition $3.5 \Gamma_{\mathfrak{a}}(N)$ and $H_{\mathfrak{a}}^{1}(N)$ are $\mathfrak{a}$-cofinite and, thus, by Proposition $3.4 \operatorname{Ext}_{R}^{i}\left(M, H_{\mathfrak{a}}^{1}(N)\right)$ and $\operatorname{Ext}_{R}^{i}\left(M, \Gamma_{\mathfrak{a}}(N)\right)$ are finite for all $i \geq 0$. Thus $H_{\mathfrak{a}}^{i}\left(M, N / \Gamma_{\mathfrak{a}}(N)\right)$ and $H_{\mathfrak{a}}^{i}\left(M, \Gamma_{\mathfrak{a}}(N)\right)$ are finite for all $i \geq 0$ which in turn shows that $H_{\mathfrak{a}}^{i}(M, N)$ is finite for all $i \geq 0$.

K.-I. Yoshida [Yo] and, D. Delfino and T. Marley [DM] proved that in the case $R$ is local and $\operatorname{dim} R / \mathfrak{a}=1, H_{\mathfrak{a}}^{i}(M)$ is $\mathfrak{a}$-cofinite for all $i \geq 0$ and all finite $R$-modules $M$. K.-I. Kawasaki [K] proved the same property of local cohomology modules in the case where $\mathfrak{a}$ is principle.

As a natural generalization to the above facts, S. Yassemi [Y2; Question 2.7] proposed a question whether the same results hold for generalized local cohomology modules. This question was affirmatively answered by K. Divaani-Aazar and R. Sazeedeh in [DS]. In the next theorem and its corollaries, we recover the main theorems of [DS] in Corollary 3.9 and Corollary 3.10; furthermore a wider class of ideals and $R$-modules are provided for which the corresponding generalized local cohomology modules are cofinite.

Theorem 3.7. Suppose that $M, N$ are finite $R$-modules with $p d(M)=t<\infty$ and $H_{\mathfrak{a}}^{i}(N)$ is $\mathfrak{a}$-cofinite for all $i \geq 0$; suppose also that the category of $\mathfrak{a}$-cofinite $R$-modules is Abelian. Then $H_{\mathfrak{a}}^{i}(M, N)$ is $\mathfrak{a}$-cofinite for all $i \geq 0$.

Proof. We prove by using induction on $t$. In the case where $t=0, M$ is a projective $R$-module; so that there exist an $R$-module $M^{\prime}$ and an integer $s$ such that $M \oplus M^{\prime} \cong R^{s}$. Hence by Lemma 2.5, for each $i \geq 0$,

$$
\begin{gathered}
H_{\mathfrak{a}}^{i}(M, N) \oplus H_{\mathfrak{a}}^{i}\left(M^{\prime}, N\right) \cong \operatorname{Hom}_{R}\left(M, H_{\mathfrak{a}}^{i}(N)\right) \oplus \operatorname{Hom}_{R}\left(M^{\prime}, H_{\mathfrak{a}}^{i}(N)\right) \\
\cong H_{R}\left(R^{s}, H_{\mathfrak{a}}^{i}(N)\right) \cong H_{\mathfrak{a}}^{i}(N)^{s} .
\end{gathered}
$$

Which implies the assertion in this case.

Suppose that $t>0$ and that the assertion holds for all finite $R$-modules with projective dimension less than $t$. Considering the exact sequence

$$
0 \rightarrow K \rightarrow R^{n} \rightarrow M \rightarrow 0
$$

where $K$ is an $R$-module and $n$ is an integer, and we obtain the long exact sequence

$$
\cdots \rightarrow H_{\mathfrak{a}}^{i-1}\left(R^{n}, N\right) \rightarrow H_{\mathfrak{a}}^{i-1}(K, N) \rightarrow H_{\mathfrak{a}}^{i}(M, N) \rightarrow H_{\mathfrak{a}}^{i}\left(R^{n}, N\right) \rightarrow H_{\mathfrak{a}}^{i}(K, N) \rightarrow \cdots
$$

By induction hypothesis $H_{\mathfrak{a}}^{i-1}\left(R^{n}, N\right), H_{\mathfrak{a}}^{i-1}(K, N), H_{\mathfrak{a}}^{i}\left(R^{n}, N\right)$ and $H_{\mathfrak{a}}^{i}(K, N)$ are $\mathfrak{a}$-cofinite while by our assumption, the category of $\mathfrak{a}$-cofinite $R$-modules is Abelian. Therefore $H_{\mathfrak{a}}^{i}(M, N)$ is $\mathfrak{a}$ cofinite as we desired.

Applying [Mel; Theorems 7.4 and 7.10] and the above theorem it is straightforward to deduce the following corollary. 
Corollary 3.8. Suppose that $\operatorname{dim} R \leq 2$ and $M, N$ are finite $R$-modules with $p d(M)<\infty$. Then $H_{\mathfrak{a}}^{i}(M, N)$ is $\mathfrak{a}$-cofinite for all $i \geq 0$.

The next two corollaries have already been proved in [DS; Theorems 2.8 and 2.9]. Here we present new proofs based on Theorem 3.7.

Corollary 3.9. Suppose that $\mathfrak{a}$ is a principle ideal. Then for finite $R$-modules $M, N$ with $p d(M)<$ $\infty, H_{\mathfrak{a}}^{i}(M, N)$ is $\mathfrak{a}$-cofinite for all $i \geq 0$.

Proof. According to Proposition 3.5 and Theorem 3.7 it is enough to prove that the category of $\mathfrak{a}$-cofinite $R$-modules is Abelian.

Let $f: L \rightarrow T$ be an $R$-homomorphism where $L, T$ are two a-cofinite $R$-modules. To prove that the category of a-cofinite $R$-modules is Abelian we need only to show that $I=i m f$ is an $\mathfrak{a}$-cofinite $R$-module.

Let $K=\operatorname{ker} f$ and $C=\operatorname{coker} f$. Considering the exact sequences

$$
0 \rightarrow I \rightarrow T \rightarrow C \rightarrow 0 \text { and } 0 \rightarrow K \rightarrow L \rightarrow I \rightarrow 0
$$

one can obtain exact sequences

$$
0 \rightarrow 0:_{I} \mathfrak{a} \rightarrow 0:_{T} \mathfrak{a} \rightarrow 0:_{C} \mathfrak{a} \text { and } K / \mathfrak{a} K \rightarrow L / \mathfrak{a} L \rightarrow I / \mathfrak{a} I \rightarrow 0 .
$$

The latter exact sequences imply that $0:_{I} \mathfrak{a}$ and $I / \mathfrak{a} I$ are finite. Therefore, by [Mel; Corollary 3.4], $I$ is $\mathfrak{a}$-cofinite as we desired.

Corollary 3.10. Suppose that $\mathfrak{p}$ is a prime ideal of a complete local ring $(R, \mathfrak{m})$ with $\operatorname{dim} R / \mathfrak{p}=1$. Then for finite $R$-modules $M, N$ with $p d(M)<\infty, H_{\mathfrak{p}}^{i}(M, N)$ is $\mathfrak{p}$-cofinite for all $i \geq 0$.

Proof. Our assumption on $\mathfrak{p}$ in conjunction with [DM; Theorem 2] imply that the category of $\mathfrak{p}$ cofinite $R$-modules is Abelian; so that this corollary is an consequence of Theorem 3.7 and the mentioned theorem of Yoshida and, Delfino and Marley.

Corollary 3.11. Suppose that $(R, \mathfrak{m})$ is a local ring such that $R / \mathfrak{a}$ is analytically irreducible (for example regular) of dimension one. Then for finite $R$-modules $M, N$ with $\operatorname{pd}(M)<\infty, H_{\mathfrak{a}}^{i}(M, N)$ is $\mathfrak{a}$-cofinite for all $i \geq 0$.

Proof. As $\widehat{R} / \widehat{\mathfrak{a}}$ is a complete local domain, by Corollary $3.10 H_{\widehat{\mathfrak{a}}}^{i}\left(M \otimes_{R} \widehat{R}, N \otimes_{R} \widehat{R}\right)$ and thus, by [DST; Lemma 2.1 (ii)], $H_{\mathfrak{a}}^{i}(M, N) \otimes_{R} \widehat{R}$ is $\widehat{\mathfrak{a}}$-cofinite for all $i \geq 0$. Now, [Mel2; Theorem 11.5(ii)] implies that $H_{\mathfrak{a}}^{i}(M, N)$ is $\mathfrak{a}$-cofinite for all $i \geq 0$.

Corollary 3.12. Let $(R, \mathfrak{m})$ be a complete local ring and $\mathfrak{a}$ be an ideal of $R$ with $\operatorname{dim} R / \mathfrak{a}=1$. Suppose also that for each $\mathfrak{a}$-cofinite $R$-module $L$ and each minimal member of $V(\mathfrak{a})$, say $\mathfrak{p}, \Gamma_{\mathfrak{p}}(L)$ and $H_{\mathfrak{p}}^{1}(L)$ are $\mathfrak{p}$-cofinite. Then $H_{\mathfrak{a}}^{i}(M, N)$ is $\mathfrak{a}$-cofinite for all $i \geq 0$ whenever $M, N$ are finite $R$-modules with $p d(M)<\infty$.

Proof. By [Mel; Proposition 7.12], our assumption on $\mathfrak{a}$ implies that the category of $\mathfrak{a}$-cofinite $R$ modules is Abelian. Now, the assertion follows from Theorem 3.7 and the mentioned theorem of Yoshida and, Delfino and Marley. 
Acknowledgments. The authors wish to thank the referee for his/her suggestions which brought improvement in the presentation of this paper.

\section{REFERENCES}

[ BS ] M. Brodmann, R.Y. Sharp, Local Cohomology, an Algebraic Introduction with Geometric Applications, Cambridge Univ. Press, Cambridge, UK, 1998.

[ DM ] D. Delfino, T. Marley, Cofinite modules and local cohomology, J. Pure Appl. Algebra 121 (1997) 45-52.

[ DS ] K. Divanni-Aazar, R. Sazeedeh, Cofiniteness of generalized local cohomology modules, Colloq. Math. 99 (2) (2004) 283-290.

[DST ] K. Divaani-Aazar, R. Sazeedeh, M. Tousi, On vanishing of generalized local cohomology modules, Algebra. Colloq. 12 (2) (2005) 213-218.

[ H ] J. Herzog, Komplexe, Aufösungen und dualitaät in der lockalen Algebra, Habilitationss chrift, Universität Regensburg. 1970.

[ K ] K.-I. Kawasaki, Cofiniteness of local cohomology modules for principal ideals, Bull. London Math. Soc. 30 (1998) 241-246.

[ M ] A. MAFi, On the associated primes of generalized local cohomology modules, Comm. Alg. 34 (2006) 2489-2494.

[Mel ] L. Melkersson, Modules cofinite with respect to an ideal, J. Algebra 285 (2005) 649-668.

[Mel2 ] L. Melkersson, Properties of cofinite modules and applications to local cohomology, Math. Proc. Cambridge Philos. Soc. 125 (1999) 417-423.

[ S ] N. Suzuki, On the generalized local cohomology and its duality, J. Math. Kyoto. Univ. 18 (1978) 71-85.

[ Y ] S. Yassemi, Generalized section functors, J. Pure. Appl. Algebra 95 (1994) 103-119.

[ Y2 ] S. Yassemi, Cofinite modules, Comm. Algebra 29 (6) (2001) 2333-2340.

[YKS ] S. Yassemi, L. Khatami, T. Sharif, Associated primes of generalized local cohomology modules, Comm. Algebra 30 (1) (2002) 327-330.

[ Yo ] K.-I. YoshidA, Cofiniteness of local cohomology modules for ideals of dimension one, Nagoya Math. J. 147 (1997) 179-191.

${ }^{1}$ Institut de mathematiques, Universite Paris 6, 4, Place Jussieu, F-75252 Paris, Cedex 05, France. And Faculty of Mathematical science and computer engineering, Teacher Training University, 599 Taleghani Avenue, 1561836314 Tehran, Iran; Fax: +98(021)77602988.

E-mail address: h_hassanzadeh@tmu.ac.ir

${ }^{2}$ Faculty of Mathematical science and computer engineering, Teacher Training University, 599 Taleghani Avenue, 1561836314 Tehran, Iran; Fax: +98(021)77602988.

E-mail address: vahidi.ar@gmail.com 